\title{
Anti-Müllerian Hormone Based Protocol Versus The Traditional Protocol For in-Vitro Fertilization/Intracytoplasmic Sperm Injection: Original A Randomized Controlled Trial
} Article

\author{
Ahmad Mahran
}

Department of Obstetrics and Gynecology, Minia University, Egypt.

\begin{abstract}
Objectives: To compare the AMH based protocol and the traditional protocol in patients undergoing IVF/ICSI. Study Design: Randomized controlled trial.

Patients and Methods: Two hundred patients candidate for IVF/ICSI were randomized intro two group; AMH-based protocol group $(n=100)$ and traditional protocol group $(n=100)$.

Results: There two groups were similar as regards the demographic features and baseline hormones. The antagonist protocol was used more frequently in the AMH group compared to the traditional protocol group (62\% vs. $19 \%, P=0.03)$. The duration of stimulation was shorter and the total dose of gonadotropins was lower in the AMH group (10.2 \pm 2.8 vs. $11.7 \pm 2.7, P=0.05$ and $2133.4 \pm 432.5$ vs. $2875.4 \pm 465.7, P=0.04$, respectively). The clinical pregnancy rate (CPR) and live birth rate were higher in the AMH group compared to the control group $(49.5 \%$ vs.30.7\%, $P=0.001$ and $43.3 \%$ vs. $23.9 \%, P=0.001$ respectively). In the AMH group, two cases were cancelled due to poor response and one case was cancelled due to moderate ovarian hyperstimulation syndrome (OHSS) with freeze all embryos, while in the traditional protocol group, five cases were cancelled due to poor response and seven cases were cancelled due to moderate / severe OHSS ( $2 \%$ vs. $5 \%, P=0.002$ and $1 \%$ vs. $7 \%, P=0.001$ respectively).

Conclusion: AMH based protocol was significantly associated with improvement of CPR and live birth rate while reducing the adverse outcomes such as OHSS and cycle cancellation rate in patients undergoing IVF/ICSI.
\end{abstract}

Submitted: 14 December 2016, Accepted: 1 February 2017.

Key Words: Anti- Müllerian Hormone, Clinical Pregnancy Rate, Cycle Cancellation, Live Birth Rate, Ovarian Hyperstimulation Syndrome.

Corresponding Author: Ahmad Mahran, MD, MRCOG, Department of Obstetrics and Gynecology, Faculty of Medicine, Minia University, Egypt., Tel.: 002-01115551073, E-mail: ezzeldin_ahmad@yahoo.com

ISSN: 2090-7265, February 2017, Vol. 7, No. 1

\section{INTRODUCTION}

Anti-Müllerian hormone (AMH) is a dimeric glycoprotein, a member of the transforming growth factor (TGF) $-b$ super family, secreted exclusively by granulose cells of primary, pre-antral and small antral follicles $(4-6 \mathrm{~mm})^{1}$.

Serum AMH level is used to assess the ovarian reserve as it has been correlated with the number of small follicles ${ }^{2}$.

Several chemical and sonographic markers have been used for the assessment of ovarian reserve. FSH levels rise while inhibin $\mathrm{B}$ levels diminish with advanced age ${ }^{3}$ (welt et al., 1997).
Ultrasound can directly assess the antral follicle count (AFC) ${ }^{4}$; However, early follicular phase measurement in young normal ovulatory women at 3-year interval revealed significant decrease in serum AMH levels, whereas serum levels of FSH and inhibin $\mathrm{B}$, besides the number of antral follicles do not change during this interval ${ }^{5}$. Thus changes in serum AMH levels occur relatively earlier than other markers. By the time FSH levels rise, cyclesbecome already irregular ${ }^{6}$. In addition, serum AMH shows a negative correlation with age but a positive correlation with AFC at ultrasound ${ }^{5,7}$. 
The role of $\mathrm{AMH}$ as a peripheral signal of the size of the growing follicle pole can be implemented in clinical practice. In patients undergoing fertility treatment, ovarian aging is characterized by decreasing ovarian responsiveness to exogenous gonadotrophin administration and poor pregnancy outcome. $\mathrm{AMH}$ can be used for identification of poor responders by assessment of their ovarian reserve before entering IVF program ${ }^{8}$.

Several studies have shown that AMH is an excellent marker of ovarian responsiveness in IVF program. Poor ovarian response defined as the number of oocytes retrieved, or as cancellation due to impaired or absent follicular growth was noticed in women with lower serum AMH levels measured in the early follicular phase (7\&9). AMH serum levels were shown to be strongly correlated with the number of antral follicles before treatment and number of oocytes retrieved upon ovarian stimulation. The predictive value of AMH for poor response is shown to be better than serum levels of $\mathrm{FSH}$, inhibin $\mathrm{B}$, and E2 and identical to $\mathrm{AFC}^{6}$. It was also shown that high serum level of AMH is associated with higher risk of cycle cancellation due to ovarian hyperstimulation syndrome $^{10}$.

In normal menstrual cycle, the early antral follicle pool remains intact throughout the follicular phase. However upon controlled ovarian hyperstimulation $(\mathrm{COH})$, all small antral follicles are stimulated to the preovulatory stage. Serum AMH measurement during FSH treatment showed significant decline at each consecutive measurement reflecting the decrease in the number of small antral follicles and providing a model to determine the relationship between AMH and follicular dynamics ${ }^{11}$.

Measurement of serum AMH levels has several advantages over other serum markers such as FSH, Inhibin $\mathrm{B}$ and E2. One single hormone measurement for $\mathrm{AMH}$ seems sufficient due to relative stability of intra-cycle serum level allowing more flexibility in the timing of measurement ${ }^{12-14}$.

We aimed to compare the AMH based protocol and the traditional protocol in terms of clinical outcome in patients undergoing IVF/ICSI.

\section{PATIENTS AND METHODS}

This is a randomized controlled trial including 200 women, who underwent IVF/ICSI treatment at Minia IVF center in the period between January 2013 and December 2015.

Inclusion criteria for the study were: a age $\leq 40$ years, b first cycle IVF/ICSI using fresh embryos and c) normal uterine cavity assessed by hysteroscopy.
Exclusion criteria were: a age $>40$ years, b) cases in whom testicular biopsies were used, c distorted uterine cavities, d hypogonadotropic hypogonadism $(\mathrm{HH})$.

Ethical approval was obtained from the institutional review board and each case gave a written informed consent before enrollment in the study.

\section{Sample size calculation:}

Sample size was calculated to prevent type II error. Clinical pregnancy rate (CPR) at the unit where the study was conducted was $10 \%$. To be of clinical significance, it was assumed that AMH based protocol should increase CPR by $50 \%$. Based on these data, we would need to study 97 patients in each arm to be able to reject the null hypothesis that the rates for study and control groups are equal in CPR with a probability of $80 \%$. The type one error probability associated with this test for the null hypothesis is 0.05 . To compensate for discontinuation, we recruited 100 patients in each arm.

\section{Randomization:}

Eligible patients who accepted to take part in the study were randomized into either study group (AMH based protocol, $\mathrm{n}=100$ ) or control group (Traditional protocol, $\mathrm{n}=100)$. Randomization was done simply using sealed envelopes. Neither patients nor doctors were blinded to the treatment assigned.

\section{Pituitary Down-regulation (DR) and Controlled Ovarian Hyperstimulation $(\mathrm{COH})$ protocols:}

Pituitary DR and $\mathrm{COH}$ were achieved using one of two protocols; traditional protocol or AMH based protocol.

\section{Traditional protocol:}

The mid-luteal long agonist protocol was used with SC administration of $0.1 \mathrm{mg}$ Decapeptyl (Decapeptyl, Ferring, Germany) starting from day 21 of the preceding cycle and continued throughout stimulation till day of HCG injection. Ovarian stimulation was achieved by IM injection of hMG starting from cycle day 2. In the antagonist protocol, ganirelix (Orgalutran, Organon, The Netherlands) $0.25 \mathrm{mg} \mathrm{SC}$ was given when a leading follicle reached $14 \mathrm{~mm}$ and continued till day of HCG. The starting dose of hMG was calculated according to patients' chronological age as shown in Table 1.

\section{AMH based protocol:}

Basal AMH level was measured in the preceding cycle. The down-regulation and the starting dose of hMG were adjusted according to AMH level as shown in Table 2. 
Ovarian follicular responses were monitored with transvaginal ultrasound. Ultrasound scanning was started on day 6 of stimulation then every other day. Step up or step down protocols was decided according to individual patients' responses. HCG injection was given (Choriomone 10,000 IU im, Choriomone, IBSA, Switzerland) when at least 3 follicles greater than $16 \mathrm{~mm}$ in diameter were detected on transvaginal ultrasound scan with leading follicle reached $18-20 \mathrm{~mm}$ in diameter. Oocyte retrieval was performed under anesthesia 36 hours after HCG administration. Insemination was performed by standard IVF or ICSI.

\section{Cycle cancellation:}

\section{Criteria for cycle cancellation in the study were:}

- Poor response: defined as $\leq 3$ follicles seen on transvaginal ultrasound scan on day 8 of $\mathrm{COH}$.

- Ovarian hyperstimulation syndrome (OHSS) leading to cycle cancellation or freeze all embryos. Cancellation was done if serum estradiol $\left(\mathrm{E}_{2}\right)$ level was $\geq 21000 \mathrm{pmol} / \mathrm{L}$ and decision for freeze all embryos was taken if E2 was between 17000 and $21000 \mathrm{pmol} / \mathrm{L}$ or number of oocytes retrieved was $>20([15])$.

\section{Biochemical assay:}

Serum samples were assayed for AMH in duplicate using an ELISA kit (Uscan), which is a sandwich enzyme immunoassay for the in vitro quantitative measurement of AMH in serum, plasma, and other biological fluids. This kit has an intra- and inter assay coefficient of variation of less than $10 \%$ and less than $12 \%$, respectively. The minimum detectable level of human AMH by this kit was typically greater than 0.046 $\mathrm{ng} / \mathrm{mL}$, with a detection range of $0.156-10 \mathrm{ng} / \mathrm{mL}$. The assay has high sensitivity and excellent specificity for detection of human AMH with no significant crossreactivity or interference.

\section{Outcome measures:}

\section{- Primary outcome measure:}

- Clinical pregnancy rate; calculated as the number of the patients with clinical pregnancy (detection of fetal heart beat with ultrasound scan) divided by the number of patients who had ET.

\section{- Secondary outcome measures:}

- Live birth rate; calculated as the ratio of the number of patients with live births divided by the number of patients who had ET.
- Cycle cancellation rate; defined as number of cycles cancelled divided by the number of cycle started.

- Multiple gestations rate; calculated as the number of patients who had multiple gestation divided by the number of patients who had clinical pregnancies.

- Miscarriage rate; calculated as the number of patients who had miscarriage divided by the number of patients who had clinical pregnancies.

Statistical methodology:

Statistical analysis was performed using Statistical Package for Social Science (SPSS Inc, Chicago) version 21 for Microsoft Windows. Data were described in terms of mean $\pm \mathrm{SD}$ (standard deviation) for continuous variables and frequencies (number of cases) and percentages for categorical data. Independent Student's t-test was used to compare quantitative variables and Mann-Whitney $U$ test was used to compare categorical data. $P<0.05$ was considered significant.

\section{RESULTS}

The study included 200 patients divided into two groups; traditional protocol group and AMH based protocol group. The two groups were similar as regards the demographic features, causes and duration of infertility and baseline hormones as shown in Table 3.

The antagonist protocol was used more frequently in the AMH-based protocol group compared to the traditional protocol group ( $62 \%$ vs. $19 \%, P=0.03)$. The duration of stimulation was shorter and the total dose of gonadotropins was lower in the AMH group $(10.2 \pm 2.8$ vs. $11.7 \pm 2.7, P=0.05$ and $2133.4 \pm$ 432.5 vs. $2875.4 \pm 465.7, P=0.04$, respectively). The total number of retrieved-oocytes was higher in the traditional group as compared with the AMH group $(13.4 \pm 5.2$ vs. $10.1 \pm 4.9, P=0.001)$. However, there was no significant difference regarding the number of mature oocytes, the number of available embryos and the number of transferred embryos between the two groups. Details of $\mathrm{COH}$ cycles are shown in Table 4.

The CPR and live birth rate were higher in the AMH group compared to the control group (49.5\% vs. $30.7 \%, P=0.001$ and $43.3 \%$ vs. $23.9 \%, P=0.001$, respectively). There was no significant difference in the multiple pregnancy and miscarriage rates between the two groups. There was one case of moderate OHSS and no cases of severe OHSS in the AMH group compared to five cases of moderate OHSS and two cases of severe OHSS in the control group ( 1 vs. $5, P=0.003$ and 0 vs. 2. $P=0.2$, respectively). In the $\mathrm{AMH}$ group, two cases were cancelled due to poor response and one case 
was cancelled due to moderate OHSS with freeze all embryos, while in the traditional protocol group, five cases were cancelled due to poor response and seven cases were cancelled due to moderate/severe OHSS ( $2 \%$ vs. $5 \%, P=0.002$ and $1 \%$ vs. $7 \%, P=0.001$, respectively).

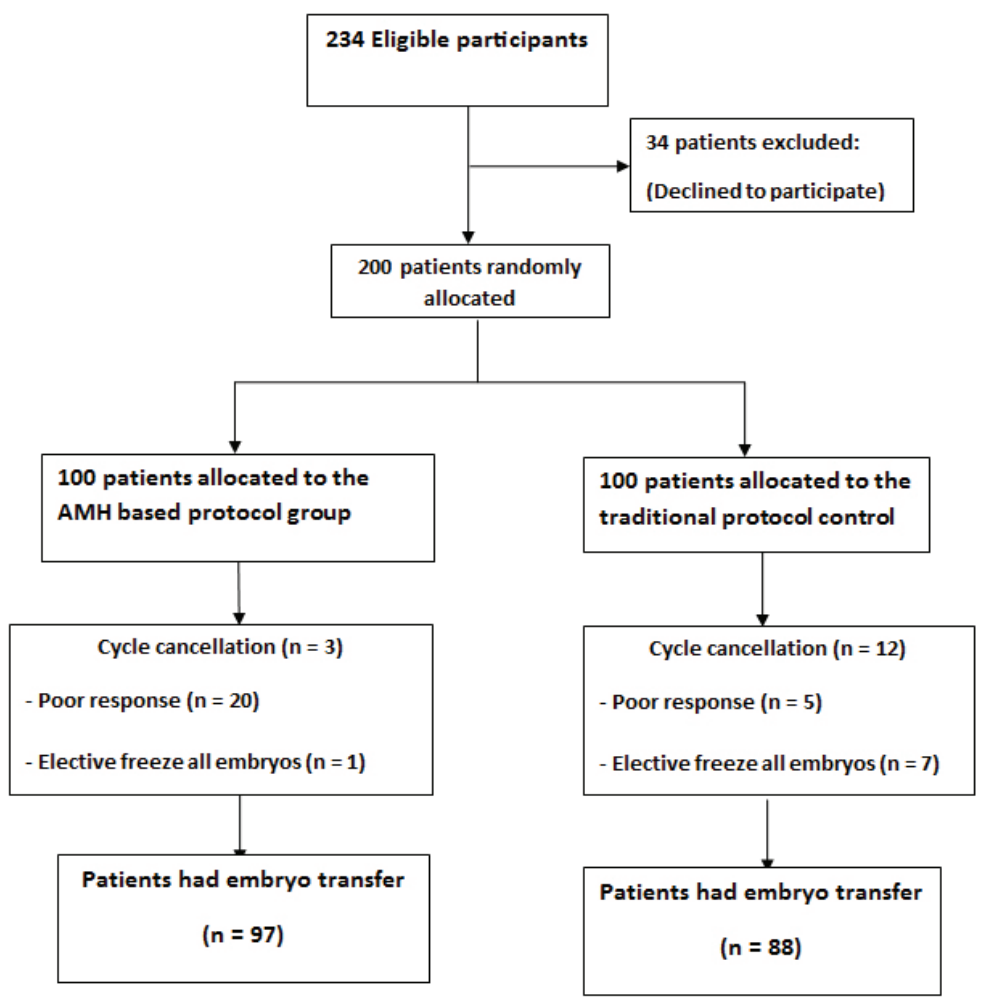

Figure 1: Study flow chart.

Table 1: Starting dose of hMG in the traditional protocol.

\begin{tabular}{lcc}
\hline Patients' chronological age & Pituitary Down-regulation protocol & Starting dose of hMG \\
\hline$<30$ years & Long agonist protocol & $225 \mathrm{IU}$ \\
\hline $30-35$ years & Long agonist protocol & $300 \mathrm{IU}$ \\
$>35$ years: & & $375 \mathrm{IU}$ \\
\hline$-\mathrm{FSH} \leq 10 \mathrm{IU} / \mathrm{L}$ & Long agonist protocol & $450 \mathrm{IU}$ \\
\hline$-\mathrm{FSH}>10 \mathrm{IU} / \mathrm{L}$ & Antagonist protocol \\
\hline
\end{tabular}

Table 2: Down-regulation protocol and starting dose of hMg in $\mathrm{AMH}$ based protocol.

\begin{tabular}{lcc}
\hline Basal AMH level & Pituitary Down-regulation protocol & Starting dose of hMG \\
\hline$<1.5 \mathrm{ng} / \mathrm{ml}:$ & Antagonist protocol & $375 \mathrm{IU}$ \\
\hline $1-1.49 \mathrm{ng} / \mathrm{ml}$ & & $450 \mathrm{IU}$ \\
\hline$<1 \mathrm{ng} / \mathrm{ml}$ & Long agonist protocol & $225 \mathrm{IU}$ \\
\hline $1.5-5 \mathrm{ng} / \mathrm{ml}$ & Antagonist protocol & $150 \mathrm{IU}$ \\
\hline$>5 \mathrm{ng} / \mathrm{ml}$ & &
\end{tabular}


Table 3: Characteristics of the study population.

\begin{tabular}{|c|c|c|c|}
\hline & $\begin{array}{c}\text { AMH based protocol } \\
(\mathrm{n}=100)\end{array}$ & $\begin{array}{c}\text { Traditional protocol } \\
\qquad(\mathrm{n}=\mathbf{1 0 0 )}\end{array}$ & $P$ value \\
\hline Age & $32.3 \pm 5.2$ & $31.4 \pm 5.1$ & 0.5 \\
\hline \multicolumn{4}{|l|}{ Infertility type: } \\
\hline - Primary & $73(73 \%)$ & $69(69 \%)$ & 0.5 \\
\hline - Secondary & $27(27 \%)$ & $31(31 \%)$ & 0.7 \\
\hline Duration of infertility & $5.3 \pm 1.5$ & $5.8 \pm 1.6$ & 0.6 \\
\hline \multicolumn{4}{|l|}{ Cause of infertility: } \\
\hline - Anovulation & 12 & 10 & 0.7 \\
\hline - Tubal factor & 7 & 5 & 0.3 \\
\hline - Male factor & 32 & 33 & 0.8 \\
\hline - unexplained & 49 & 52 & 0.7 \\
\hline $\operatorname{BMI}\left(\mathrm{kg} / \mathrm{m}^{2}\right)$ & $28.1 \pm 3.8$ & $27.7 \pm 4.1$ & 0.6 \\
\hline $\mathrm{FSH}(\mathrm{IU} / \mathrm{L})$ & $7.7 \pm 1.9$ & $7.5 \pm 1.9$ & 0.7 \\
\hline LH (IU/L) & $5.3 \pm 1.5$ & $5.4 \pm 1.5$ & 0.7 \\
\hline $\mathrm{AMH}(\mathrm{ng} / \mathrm{ml})$ & $2.8 \pm 0.8$ & & \\
\hline
\end{tabular}

Data is presented as mean \pm SD or frequency and percentages.

Table 4: Details of $\mathrm{COH}$ cycles.

\begin{tabular}{|c|c|c|c|}
\hline & $\begin{array}{l}\text { AMH based protocol } \\
(\mathbf{n}=100)\end{array}$ & $\begin{array}{l}\text { Traditional protocol } \\
\qquad(\mathbf{n}=\mathbf{1 0 0})\end{array}$ & $P$ value \\
\hline \multicolumn{4}{|l|}{ Down-regulation protocol: } \\
\hline - Long agonist protocol & $38(38 \%)$ & $81(81 \%)$ & $0.03^{*}$ \\
\hline - Antagonist protocol & $62(62 \%)$ & $19(19 \%)$ & $0.02^{*}$ \\
\hline Duration of stimulation (days) & $10.2 \pm 2.8$ & $11.7 \pm 2.7$ & $0.05^{*}$ \\
\hline Dose of gonadotropins & $2133.4 \pm 432.5$ & $2875.4 \pm 465.7$ & $0.04^{*}$ \\
\hline Total no. of retrieved oocytes & $10.1 \pm 4.9$ & $13.4 \pm 5.2$ & $0.001^{*}$ \\
\hline No. of mature oocytes & $8.8 \pm 2.5$ & $9.3 \pm 2.9$ & 0.6 \\
\hline No. of available embryos & $7.2 \pm 1.4$ & $7.4 \pm 1.6$ & 0.8 \\
\hline No. of embryos transferred: & 7 & 5 & 0.7 \\
\hline - One & 7 & 5 & 0.7 \\
\hline - Two & 65 & 76 & 0.2 \\
\hline - Three & 10 & 11 & 0.8 \\
\hline$->$ three & 6 & 5 & 0.7 \\
\hline
\end{tabular}

Data is presented as mean $\pm \mathrm{SD}$ or frequency and percentages.

"statistically significant. 
Table 5: Cycle outcome in the study population.

\begin{tabular}{|c|c|c|c|}
\hline & $\begin{array}{l}\text { AMH based protocol } \\
\qquad(\mathrm{n}=100)\end{array}$ & $\begin{array}{c}\text { Traditional protocol } \\
\qquad(\mathrm{n}=\mathbf{1 0 0 )}\end{array}$ & $P$ value \\
\hline Clinical pregnancy rate (CPR) & $48 / 97(49.5 \%)$ & $27 / 88(30.7 \%)$ & $0.001^{*}$ \\
\hline Live birth rate & 42/97 (43.3\%) & $21 / 88(23.9 \%)$ & $0.001^{*}$ \\
\hline Multiple pregnancy rate & $5 / 48(10.4 \%)$ & $4 / 27(14.8 \%)$ & 0.3 \\
\hline Miscarriage rate & $6 / 48(12.5 \%)$ & $5 / 27(18.5 \%)$ & 0.2 \\
\hline \multicolumn{4}{|l|}{ OHSS: } \\
\hline - Mild & $5(5 \%)$ & $7(7 \%)$ & 0.5 \\
\hline - Moderate & $1(1 \%)$ & $5(5 \%)$ & $0.003^{*}$ \\
\hline - Severe & $0(0 \%)$ & $2(2 \%)$ & $0.02^{*}$ \\
\hline \multicolumn{4}{|l|}{ Cycle cancellation: } \\
\hline - Poor response & $2(2 \%)$ & $5(5 \%)$ & $0.002^{*}$ \\
\hline - Elective freeze all embryos & $1(1 \%)$ & $7(7 \%)$ & $0.001^{*}$ \\
\hline
\end{tabular}

Data is presented as mean $\pm \mathrm{SD}$ or frequency and percentages.

"statistically significant.

\section{DISCUSSION}

Until now, there is a general consensus that $\mathrm{AMH}$ is an excellent marker for ovarian reserve ${ }^{16}$. Several studies have evaluated the use of AMH to predict ovarian response to stimulation in ART. AMH shown to have a good predictive value for poor response to stimulation in some studies (15\&17); while other studies did not support this conclusion (18\&19). The predictive value of $\mathrm{AMH}$ for over response is agreed upon by different studies $(15,19 \& 20)$. There is no consensus about the association of AMH levels with pregnancy and live birth rates in IVF/ICSI programs ${ }^{19}{ }^{-22}$. Individualization of $\mathrm{COH}$ according to $\mathrm{AMH}$ levels has been tried in previous studies with no consensus about the cut-off levels for AMH (23\&24).

In this study, we tried to tailor the down-regulation and $\mathrm{COH}$ protocols according to $\mathrm{AMH}$ levels. Upon patients' responses to treatment in the same center, we selected the cut-off levels where the study was conducted. We compared the clinical outcome of AMHbased protocol with the traditional protocol according to the patients' chronological age and basal FSH levels. In the current study, the use of AMH-based protocol triggered more implication of the antagonist protocol which is shown previously to improve the outcome in patients at both low and high extremes of ovarian reserve $^{25}$

In a UK tertiary care unit, Yates et al. included 769 women in their first IVF, of them 346 women underwent the conventional stimulation protocol (using chronological age and FSH as a guide for treatment), while 423 women treated with AMH tailored protocol. They reported a pregnancy rate of $27.7 \%$ with a live birth rate of $23.9 \%$ per cycle started in the AMH-tailored protocol group compared to $17.9 \%$ and $15.9 \%$, respectively in the conventional protocol group. Cycle cancellation rate and OHSS were significantly lower in the AMH-tailored protocol group. In addition, they addressed the cost for $\mathrm{COH}$ fertility drugs and treatment of OHSS, whereas. the AMH-tailored protocol was more cost-effective than the conventional protocol. The limitations of their study were the retrospective nature, besides, the two protocols were not carried out in parallel (i.e. were not used in the same period $)^{24}$.

In our research, $\mathrm{AMH}$-based protocol was associated with improvement in CPR and live birth rate compared to the traditional protocol. "Moreover, the AMH based protocol was shown to reduce the adverse outcome as OHSS and cycle cancellation either due to poor response or freeze all embryos as a result of OHSS". Noteworthy, the duration of $\mathrm{COH}$ and the dose of gonadotropins were significantly reduced, which give the AMHbased protocol the potential for a cost-effective effect especially when counting the cost of treatment of OHSS and the additional cost for embryo-freezing.

The prospective nature and reporting important clinical outcome with potential implication for clinical practice are the points of strength in this study are the.

The limitation of our study is non-reporting the cost effectiveness of the AMH protocol, whereas the cost of treatment of cases of OHSS was difficult to be estimated since most of our cases were treated at governmentally funded general hospitals. 
In conclusion, compared to the traditional protocol, the AMH based protocol significantly associated with improvement of CPR and live birth rate while reducing the adverse outcomes such as OHSS and cycle cancellation rate.

Based on our findings, the AMH based protocol may be used in routine IVF practice.

\section{ACKNOWLEDGMENT}

The author is quite grateful to the entire medical, laboratory and nursing staff at Minia IVF center for their help throughout the research work.

\section{CONFLICT OF INTEREST}

The author declares no conflict of interest related to this study.

\section{REFERENCES}

1. Kuroda T, Lee MM, Haqq CM, Powell DM, Manganaro TF, Donahoe PK. Müllerian inhibiting substance ontogeny and its modulation by follicle-stimulating hormone in the rat testes. Endocrinology1990; 127:1825-1832.

2. Weenen C, Laven JS, von Bergh AR, et al. AntiMullerian hormone expression pattern in the human ovary: potential implications for initial and cyclic follicle recruitment. Mol Hum Reprod 2004;10:77-83.

3. Welt CK, McNicholl DJ, Taylor AE, Hall JE. Female reproductive aging is marked by decreased secretion of dimeric inhibin. J Clin Endocrinol Metab 1999;84:105-11.

4. Pache TD, Wladimiroff JW, de Jong FH, Hop WC, Fauser BCJM.Growth patterns of nondominant ovarian follicles during the normal menstrual cycle. Fertil Steril 1990; 54: 638-642.

5. A de Vet, Laven JS, de Jong FH, Themmen APN\& Fauser BC. Antimullerian hormone serum levels: a putative marker for ovarian aging. Fertility and Sterilit y2002; 77: 357-362.

6. Burger HG, Dudley EC, Hopper JL, Groome N, Guthrie JR, Green A \& Dennerstein L Prospectively measured levels of serum follicle-stimulating hormone, estradiol, and the dimeric inhibins during the menopausal transition in a population-based cohort of women. Journal of Clinical Endocrinology and Metabolism 1999; 84 :025-4030.
7. van Rooij IA, Broekmans FJ, te Velde ER, Fauser BC, Bancsi LF, de Jong FH \& Themmen APN. Serum anti-Mullerian hormone levels: a novel measure of ovarian reserve. Human Reproduction 2002; 17: 3065-3071.

8. Visser JA, de Jong FH, Laven JSE, Themmen APN. Anti-Mullerian hormone: a new marker for ovarian function. Reproduction 2006; 131:1-9.

9. Seifer DB, MacLaughlin DT, Christian BP, Feng B \& Shelden RM Early follicular serum mullerianinhibiting substance levels are associated with ovarian response during assisted reproductive technology cycles. Fertility and Sterilty 2002; $77: 468-471$.

10. La Marca A, Giulini S, Tirelli A , Bertucci E, Marsella T, Xella S and Volpe A. Anti-Müllerian hormone measurement on any day of themenstrual cycle strongly predicts ovarian response in assisted reproductive technology. Human Reproduction 2007;.22, No.3: 766-771.

11. Fanchin R, Schonauer LM, Righini C, Frydman N, Frydman R, Taieb J. Serum anti-Mullerian hormone dynamics during controlled ovarian hyperstimulation. Human Reproduction 2003a; 18 :328-332.

12. Fanchin R, Taieb J, Lozano DH, Ducot B, Frydman $\mathrm{R}$, Bouyer J. High reproducibility of serum antiMullerian hormone measurements suggests a multistaged follicular secretion and strengthens its role in the assessment of ovarian follicular status. Human Reproduction 2005a; 20: 923-927.

13. Tsepelidis S, Devreker F, Demeestere I, Flahaut A, Gervy Ch, Englert Y. Stable serum levels of antiMüllerian hormone during the menstrual cycle: a prospective study in normo-ovulatory women. Hum Reprod 2007; 22:1837-1840.

14. Van Disseldorp J, Lambalk CB, Kwee J, Looman CWN, Eijkemans MJC, Fauser BC, Broekmans FJ. Comparison of inter- and intra-cycle variability of anti-Mu"llerian hormone and antral follicle counts. Human Reprod 2010;25:221-227.

15. Nardo LG, Gelbaya TA, Wilkinson H, Roberts SA, Yates A, Pemberton P, Laing I. Circulating basal anti$\mathrm{Mu}$ "llerian hormone levels as a predictor of ovarian response in women undergoing ovarian stimulation for in-vitro fertilisation. Fertil Steril 2009;92:1586-1593.

16. Aboulghar M. Anti-mullerian hormone in the management of infertility. MEFS 2014;19: 1-7. 
17. Gnoth C, Schuring AN, Friol K, Tigges J, Mallmann P, Godehardt E. Relevance of anti-Mu"llerian hormone measurement in a routine IVF program. Hum Reprod 2008;23:1359-1365.

18. Broekmans FJ, Kwee J, Hendriks DJ, Mol BW, Lambalk CB. A systematic review of tests predicting ovarian reserve and IVF outcome. Hum Reprod Update 2006;12:685-718.

19. Broer SL, Mol B, Dolleman M, Fauser B C, Broekmans FJ. The role of anti-Müllerian hormone assessment in assisted reproductive technology outcome. Curr Opin Obstet Gynecol 2010; 22:193-201.

20. Broer SL, Dolleman M, Opmeer BC, Fauser $\mathrm{BC}, \mathrm{Mol} \mathrm{BW}$, Broekmans FJ. AMH and AFC as predictors of excessive response in controlled ovarian hyperstimulation: a meta-analysis. Hum Reprod Update 2011;17:46-54.
21. Nelson SM, Yates RW, Fleming R. Serum antiMullerian hormone and FSH: prediction of live birth and extremes of response in stimulated cycles-implications for individualisation of therapy. Human Reprod 2007;22:2414-2421.

22. NakhudaGS, Douglas NC, ThorntonMH, Guarnaccia MM, Lobo R, Sauer MV. Anti-Mu“llerian hormone testing is useful for individualization of stimulation protocols in oocyte donors. Reprod Biomed Online 2010;20:42-47.

23. Nelson SM, Yates RW, Lyall H, Jamieson M, Traynor I, Gaudoin M, Mitchell P, Ambrose P, Fleming R. Anti$\mathrm{Mu}$ "llerian hormone-based approach to controlled ovarian stimulation for assisted conception. Human Reprod 2009;24:867-875.

24. Yates AP, Rustamov O, Roberts SA, Lim HYN, Pemberton PW, Smith A, Nardo LG. Anti-Mullerian hormone-tailored stimulatio 